\title{
CONOCIMIENTOS Y ACTITUDES DE LOS ESTUDIANTES UNIVERSITARIOS COSTARRICENSES CON RESPECTO AL VIH/SIDA ${ }^{1}$
}

\section{KNOWLEDGE AND ATTITUDES OF UNIVERSITY STUDENTS OF COSTA RICA ABOUT HIVIAIDS}

\author{
Pilar Salas Chaves * \\ Liseth Taylor Castillo ** \\ Marilyn Quesada Cambronero *** \\ Ana Cristina Garita Garita **** \\ Dennis León Alán ${ }^{* * * * *}$
}

\section{RESUMEN}

\begin{abstract}
Se realizó una encuesta auto-administrada y anónima a estudiantes matriculados en la Universidad de Costa Rica con el objetivo de identificar el nivel de conocimiento acerca del VIH-sida. Se logró recolectar un total de 497 cuestionarios durante el ciclo lectivo del año 2008. Los encuestados consideraron de alto riesgo los mecanismos de transmisión del VIH por medio de jeringas, agujas contaminadas y las relaciones sexuales con una persona infectada sin usar preservativos.

Los resultados demuestran que los estudiantes obtuvieron una nota de 65,5 y no se encontraron diferencias en las calificaciones obtenidas por los entrevistados, ni por sexo ni por edad. Se recomienda un programa educativo sobre prevención de las infecciones de transmisión sexual y el VIH/sida.
\end{abstract}

PALABRAS CLAVE: COSTA RICA * VIH * JUVENTUD * CONOCIMIENTOS * CAMPAÑAS DE PREVENCIÓN

Este proyecto recibió apoyo financiero por parte de la Vicerrectoría de Investigación a través del proyecto 803-A8-318, de la Vicerrectoría de Acción Social a través del proyecto ED-1839, de la Facultad de Microbiología y de la Oficina de Bienestar y Salud de la Universidad de Costa Rica. Facultad de Microbiología, Universidad de la Costa Rica (UCR) maria.salas@ucr.ac.cr

Facultad de Microbiología, Universidad de Costa Rica (UCR)

mayra.taylor@ucr.ac.cr

\footnotetext{
*** Oficina de Bienestar y Salud, Universidad de Costa Rica (UCR) mqc665@yahoo.es

**** Oficina de Bienestar y Salud, Universidad de Costa Rica (UCR) cristina.garitagarita@ucr.ac.cr

***** Facultad de Microbiología, Universidad de Costa Rica (UCR) denleoncr@yahoo.com
} 


\title{
ABSTRACT
}

\begin{abstract}
An anonymous survey was conducted to students enrolled in the University of Costa Rica in order to identify the level of knowledge about HIV-aids. It was managed to collect a total 497 questionnaires, during the school year 2008. Respondents considered high-risk mechanisms of transmission of HIV through needle, contaminated needles and sexual intercourse with an infected person without the use of condoms. The results show that students obtained a note of 65,5 and we found no differences in the qualifications obtained by the interviewees, by sex or age. It recommends an education program on prevention of HIV-aids.
\end{abstract}

KEYWORDS: COSTA RICA * HIV * YOUTH * KNOWLEDGE * PREVENTION CAMPAIGNS

\section{INTRODUCCIÓN}

El Síndrome de Inmunodeficiencia Adquirida (sida), es la forma en que se manifiesta la infección por el virus de la inmunodeficiencia humana (VIH). La rápida diseminación del virus, sus secuelas a nivel de salud personal, problemas sexuales y de salud reproductiva, así como, todos los problemas de estigma y discriminación que surgieron en su entorno, han hecho que sea una de las infecciones con mayor impacto a nivel mundial (UNAIDS, 2006).

De acuerdo con el reporte correspondiente al año 2007, del programa conjunto de las Naciones Unidas sobre la infección por VIH/ sida (ONUSIDA) y de la Organización Mundial de la Salud (OMS), la prevalencia mundial de la infección por VIH en la población adulta ha tendido a estabilizarse siendo de $0,8 \%$, pero el número total de infectados sigue en aumento, debido a la acumulación continua de nuevas infecciones con períodos más prolongados de sobrevida, medidos en una población general en constante crecimiento. En el año 2007, el número estimado de infectados por VIH en el mundo alcanzó 33,2 millones de personas (ONUSIDA, 2007: 7).

En el caso de Costa Rica, se estima que la tasa de incidencia es de alrededor del 0,6\%, sin tomar en cuenta el subregistro, lo que muestra una situación favorable a nivel epidemiológico (ONUSIDA y OMS, 1997). La principal vía de transmisión es sexual, por lo que es necesario realizar campañas para poder disminuir esta tasa de incidencia o al menos mantenerla.

La situación del VIH/sida en Costa Rica es considerada de baja prevalencia. Tiene una tendencia a la feminización y está ubicada en las zonas urbanas de la gran área metropolitana (Ministerio de Salud, Consejo Nacional de Atención Integral del VIH/sida, Organización Panamericana de la Salud, oficina regional de la OMS, Programa Conjunto de las Naciones Unidas sobre el VIH/sida. La situación del VIH/ sida en Costa Rica, 2004).

En Costa Rica se detectó a la primera persona infectada, en un grupo de pacientes tratados con sangre o hemoderivados. Fue a partir de 1985, cuando se reportaron los primeros casos de hombres que tienen sexo con otros hombres (HSH) (44,3\%), entre personas heterosexuales $(24,8 \%)$ y los bisexuales $(15,5 \%)$ (Víquez, 1997).

Hasta el año 1988, se reportó la primera mujer infectada por el VIH. Esto contribuyó a que se estigmatizara y relacionara al sida a ciertos grupos poblacionales y consecuentemente, se subestimara la epidemia entre los heterosexuales. En 1992, la razón hombre-mujer era de 12,9 a 1 y para el año 2005 pasó a 5,4 casos nuevos de sida del sexo masculino por cada nuevo caso del sexo femenino (Espinoza, 2006: 36). Esto demuestra que las mujeres se están infectando como consecuencia de las relaciones sexuales con su pareja (Espinoza, 2006: 36).

El sida es una infección de transmisión sexual, lo que ha llevado a los expertos a considerar que la modificación del comportamiento sexual es un elemento fundamental para su prevención y control. Experiencias en otros países han demostrado que la participación multidisciplinaria ha logrado mayores éxitos mediante campañas de 
prevención, información y educación, sobre todo en la población joven, considerándose que estas medidas son el medio que los llevará a evitar prácticas de riesgo (Pérez, 2002).

En el contagio del VIH, el comportamiento de las personas tiene un papel determinante $y$ la prevención consiste precisamente en el abandono de los comportamientos de riesgo junto con la adopción y mantenimiento de comportamientos preventivos (Plan Estratégico Nacional para la Prevención, Atención y Asistencia del VIH/sida, Ministerio de Salud, 2006).

El presente estudio tuvo como objetivo identificar el nivel de conocimientos de las y los estudiantes universitarios costarricenses con respecto al VIH/sida y contribuir con información que le permita a las autoridades universitarias y de salud del país, la realización de campañas de prevención en poblaciones específicas.

\section{METODOLOGÍA}

Se realizó una encuesta auto-administrada y anónima en la población estudiantil, matriculada en el año 2008, en las diferentes sedes que tiene la Universidad de Costa Rica en todo el país.

Se recolectaron un total de 497 cuestionarios, número que está dentro de lo admisible en encuestas sociales, que buscan evaluar el nivel de conocimiento $y$ actitudes de las personas entrevistadas y no la estimación específica de determinados parámetros.

Con el propósito de obtener un puntaje para evaluar los conocimientos de los estudiantes, se creó un índice con la suma de las respuestas correctas de ocho preguntas en total y se obtuvo una nota, según sexo y edad de los entrevistados de la siguiente manera: se asignó un valor de uno a cada respuesta correcta, se sumaron todas ellas y se dividió entre el total de preguntas de conocimiento.

\section{RESULTADOS}

La mayoría de los estudiantes, tanto hombres como mujeres, se encontraban entre los 19 y los 22 años de edad, siendo el 55\% mujeres y el $45 \%$ hombres. En cuanto a su estado conyugal, la mayoría eran solteros(as) y los y las que trabajaban oscilaban en el grupo de 29 años o más.

En cuanto a qué estudiaban, la mayoría se agruparon en el área de Ciencias Sociales. Cabe mencionar que esta área agrupa varias facultades como son: Ciencias Sociales, Ciencias Económicas, Educación, Derecho; le sigue en orden de magnitud el área de Artes y Letras y en tercer lugar, el área de Ingeniería. Los universitarios tuvieron una percepción correcta al considerar como de alto riesgo los mecanismos de contagio por medio de jeringas, agujas contaminadas y las relaciones sexuales con una persona infectada sin usar preservativo (ver anexo 1).

Con respecto al riesgo de infección por la práctica del sexo oral, se muestra que el 49,5\% de las y los encuestados consideró que el contagio es de regular y bajo riesgo.

Se observa que un porcentaje importante de las $y$ los estudiantes respondieron correctamente a diferentes planteamientos sobre conocimientos (ver anexo 2). El 33,9\% opinó que no hay riesgo de infección por la práctica del sexo anal sin protección, por lo se deben reforzar las formas de transmisión del VIH especialmente en personas que tienen relaciones sexuales anales.

Se plantean ciertas situaciones sobre prejuicios relacionados con la transmisión del virus siendo las mujeres las que se asocian a respuestas de regular o alto riesgo (ver anexo 3). Únicamente para tres situaciones los hombres indicaron una mayor proporción de respuestas con riesgo alto o regular; estas son:

a) Jugar con niño que tiene sida

b) Picadura de mosquito

c) Tener relaciones sexuales con una pareja fiel no infectada

El anexo 4 representa el nivel de conocimientos que tenían las y los estudiantes respecto al VIH/sida, en el cual se utilizó el índice creado según se describe en la metodología.

En este cuadro se presenta el valor de la mediana y el promedio de este índice o la nota de conocimientos $(65,5)$ y no se muestran diferencias en las calificaciones obtenidas por los entrevistados, ni por sexo, ni por edad. Este 
índice se obtuvo por la suma de las respuestas correctas, ocho preguntas en total, asignándole un valor de uno a cada respuesta correcta y se dividió entre el total de preguntas.

\section{DISCUSIÓN}

Los hallazgos generados en la encuesta permitieron analizar el índice de conocimientos sobre el VIH/sida, mecanismos de transmisión, medios de prevención, nivel de riesgo de contagio y estereotipos en la población estudiantil de la Universidad de Costa Rica. Los datos generados permiten concluir que las y los encuestados tienen un conocimiento insuficiente, ya que estos jóvenes obtuvieron una calificación inferior a 70 en un examen sobre conocimientos del tema. Cabe resaltar el interés manifestado por los y las estudiantes en recibir información sobre el VIH en forma general y la necesidad de contar con un mayor acceso a la información, con el fin de conocer todo lo relacionado con el VIH y el sida de una manera expedita, confiable $y$ veraz.

A finales de 1987, la Asociación Demográfica Costarricense realizó una encuesta denominada "Investigación Integral 1987", a nivel nacional, en la cual se aplicó un módulo de 15 preguntas sobre el sida a una muestra nacional de 1222 mujeres en edad fértil y tuvo como objetivo medir el nivel de conocimiento básico con respecto al sida, sus formas de transmisión y de prevención. En esta encuesta se encontró que el conocimiento general de la enfermedad alcanzaba porcentajes superiores a 70\% (Madrigal, 1988), lo cual es mayor a lo encontrado en este estudio.

Una encuesta previa realizada en 1988, en jóvenes universitarios de la Universidad de Costa Rica, tuvo como objetivo investigar el nivel de conocimientos, la actitud y el cambio en los hábitos sexuales, relacionados con el sida. Entre los resultados se encontró que los universitarios tenían conciencia de que el sida elimina las defensas naturales del cuerpo humano contra las enfermedades (96,3\%), puede atacar a personas de ambos sexos $(98,4 \%)$ y que no hay una vacuna disponible (85,7\%). El estudio demostró un conocimiento alto con respecto al sida en los universitarios en ese momento (Madrigal, 1988: 92). Se encontró además que existía una marcada desconfianza por el intercambio de sustancias corporales como saliva, heces, semen y sangre.

Los estudiantes opinaron que para evitar la propagación del sida, el examen debería ser obligatorio para trabajadores del sexo, marinos, extranjeros, personas que han tenido relaciones sexuales con personas contagiadas, a turistas y estudiantes extranjeros (Madrigal, 1988: 95).

En 1987, se llevó a cabo una campaña educativa como parte de la lucha contra el VIH/ sida, con el esfuerzo conjunto de la Comisión Nacional de sida, el Ministerio de Salud, los medios de comunicación y la empresa privada, especialmente las agencias de publicidad. Los buenos resultados encontrados en la encuesta realizada en 1988, previamente discutida, se pueden atribuir al éxito de esta campaña, las bajas notas obtenidas en el presente estudio demuestran que el efecto de una buena campaña educativa se pierde con el tiempo si la misma no es sostenida.

En el presente estudio, un 95\% de los universitarios reportaron que el sida es sexualmente transmitido $y$ que puede contagiarse por el uso de jeringas y agujas contaminadas. Estos resultados coinciden con lo reportado por Vera-Gamboa y colaboradores, en donde se pudo determinar que el 98\% de los encuestados, presentaron conocimientos adecuados y suficientes sobre el sida (2006: 159).

Existen algunos estudios en México sobre conocimientos del VIH/sida en adolescentes y jóvenes del área urbana, uno de esos estudios reportó niveles altos de conocimientos en los estratos con mayores recursos económicos que no siempre tienen correspondencia con prácticas saludables ni con la modificación de comportamientos de riesgo (Caballero y Villaseñor, 2003: 113).

En este estudio, se detectó además que el conocimiento con respecto al uso del preservativo como método de protección fue acertado entre el 75\% y $80 \%$ de los entrevistados. La Primera Encuesta Nacional de Juventud realizada en Costa Rica en el 2008, revela que el uso del preservativo es reconocido como un método anticonceptivo en la mayoría de los casos, pero 
cuando se indaga acerca de la protección de las enfermedades de transmisión sexual y el VIH su frecuencia disminuye (Consejo Nacional de la Política de la Persona Joven. Primera Encuesta Nacional de Juventud, 2008).

Con respecto al riesgo que implica la práctica del sexo oral, los entrevistados demostraron porcentajes muy divididos. Estos resultados son similares a lo encontrado por Trajman $y$ colaboradores, en donde los estudiantes consideran que la transmisión del virus a través de esta vía es de bajo riesgo (2003: 8). Igualmente, el trabajo de Vera-Gamboa y colaboradores, señala que la quinta parte de los entrevistados no consideró el sexo oral como mecanismo de transmisión (2006: 160).

En general, las mujeres presentaron porcentajes más altos en cuanto a los conocimientos sobre los mecanismos de transmisión y la prevención, más bajos con respecto a las situaciones de bajo riesgo. Estos resultados concuerdan con el trabajo realizado por Al-Jabri y colaboradores, donde la mayoría de los estudiantes mostraron un conocimiento razonable de la transmisión, comportamiento de riesgo y prevención, pero tienen una falsa percepción de la enfermedad (2003: 274). En un estudio similar llevado a cabo por Raza $y$ colaboradores, encontraron que los estudiantes consideran que el VIH puede ser transmitido por contacto casual o por la picadura de un mosquito (1998: 180).

Según Vera y colaboradores (2006), la idea de otras formas de transmisión va más en relación con la actitud y al temor ante esa enfermedad y coincide con el estudio de Egger, en estudiantes de secundaria en Nicaragua (Egger; Ferrie y Gorter, 1994: 367) (Sekirime; Tamale y Lule, 2001: 20).

Los programas orientados a inducir cambios de comportamiento que se realizan en muchos países, incluyen información básica sobre el virus, evaluación del riesgo personal, capacidad para negociar el uso del preservativo con las parejas, entre otros. Este conocimiento e información son las primeras líneas de defensa para los jóvenes que son los más amenazados, ya que mundialmente representan la mitad de los casos nuevos de VIH (Macchi y Benitez,
2008: 208). Estos programas de prevención no han sido lo suficientemente fuertes y efectivos para integrar las áreas de prevención, tratamiento y atención al VIH (Macchi y Benitez, 2008: 215).

Para ello, se deben incluir temas de educación sexual en los planes de estudio de los colegios y universidades para aumentar el conocimiento correcto $y$ la prevención de las enfermedades de transmisión sexual, incluida el sida (Raza; Afifi y Choudhry, 1998:180).

A pesar de que el comportamiento está directamente asociado al riesgo o no de infectarse con el VIH, no es posible tener un comportamiento de protección si los individuos no tienen el conocimiento de prevención.

En el contagio del VIH, tiene un papel determinante el comportamiento de las personas $y$ la prevención consiste precisamente en el abandono de los comportamientos de riesgo, junto con la adopción y mantenimiento de comportamientos preventivos.

Por lo tanto, como una de las estrategias de la atención integral en salud para la población estudiantil en general y retomando el modelo de salud en Costa Rica, el cual contempla la prevención mediante la educación, se considera importante la implementación de un programa educativo sobre prevención de las infecciones de transmisión sexual y el VIH/sida en la comunidad universitaria costarricense que tome como base los puntos débiles encontrados.

\section{REFERENCIAS BIBLIOGRÁFICAS}

Al-Jabri, A.A. y Al-Abri, J.H. "Knowledge and attitudes of undergraduate medical and non-medical students in Sultan Cabos University toward acquired immune deficiency syndrome”. Saudi. Med. J. 24 (3). 2003: 273-7.

Caballero-Hoyos, J. R. y Villaseñor-Sierra, A. "Conocimientos sobre el VIH/sida en adolescentes urbanos: consenso cultural de dudas e incertidumbres". Sal. Pub. Mex. 45 (1). 2003: 108-114.

Consejo Nacional de la Política de la Persona Joven. Primera Encuesta Nacional de Juventud. Ministerio de Cultura, 2008. 
Egger, M. et ál. "Conocimientos, actitudes y prácticas acerca del sida en estudiantes de escuelas secundarias de Managua". Bol. Of. Sanit. Panam. 117. 1994: 12-21.

Espinoza, A. "Tendencias y factores de riesgo del VIH/sida en Costa Rica, 1993 al 2001". Rev. Cos. Salud. Pub. 15 (9). 2006: 35-43.

Macchi, M.L. et ál. "Conocimientos, actitudes y prácticas acerca del VIH/sida en jóvenes a nivel medio de educación, del área metropolitana, Paraguay". Rev. Chil. Pediatr. 79 (2). 2008: 206-217.

Madrigal, J. "La población universitaria y el sida". Rev. Cost. Cienc. Médicas 3(9). 1988: 91-105.

Madrigal, J. "Sida. Conocimiento básico de la mujer en Costa Rica". Revista Costarricense de Ciencias Médicas 3(9). 1988: 81-90.

Ministerio de Salud, Consejo Nacional de Atención Integral del VIH/sida. Organización Panamericana de la Salud, oficina regional de la OMS, Programa Conjunto de las Naciones Unidas sobre el VIH/sida, La situación del VIH/sida en Costa Rica. 2004.

Ministerio de Salud. Plan Estratégico Nacional para la Prevención, Atención y Asistencia del VIH/sida. 2006.

ONUSIDA. "Estadísticas mundiales VIH/sida". Documentos ONUSIDA y OMS. 1997.
Pérez, C. "Estrategias de prevención del VIH/ sida, Biomedia”. XIV Conferencia Internacional Sida. Barcelona, 2002.

Programa conjunto de las Naciones Unidas sobre el VIH/sida (ONUSIDA) y la Organización Mundial de la Salud (OMS). 2007.

Raza, M. I. et ál. "Knowledge, attitude and behaviour toward AIDS among educated youth in Lahore, Pakistan". J. Pak. Med. Assoc. 48 (6). June 1998: 179-82.

Sekirime, W. K. et ál. "Knowledge, attitude and practice about sexually transmitted diseases among university students in Kampala". Afr. Health Sci. 1. Aug. 2001: 16-22.

Trajman, A. et ál. "Knowledge about STD/AIDS and sexual behaviour among high school students in Rio de Janeiro, Brazil". Cad. Saúde Publica 19 (1). 2003: 1-11.

UNAIDS. AIDS Epidemics update: special report on HIV/AIDS. Geneva, Switzerland. December 2006.

Vera-Gamboa, L.; Sánchez-Magallón, F. y Gongora-Biachi, R.A. "Conocimientos $y$ percepción de riesgo sobre el sida en estudiantes de bachillerato de una universidad pública de Yucatán, México: un abordaje cuantitativo-cualitativo". Rev. Biomed. 17. 2006: 57-168.

Víquez, A.; Elizondo, J.; Cercone, J. y Jaramillo, O. VIH/sida en Costa Rica: situación actual y proyecciones. SIDALAC, 1997: 71pp. 
ANEXOS

\author{
CUADRO 1
}

UNIVERSIDAD DE COSTA RICA: OPINIÓN DE LOS ESTUDIANTES CON RESPECTO AL GRADO DE RIESGO DE CONTAGIARSE DEL VIRUS VIH ANTE DISTINTAS SITUACIONES, 2008

\begin{tabular}{|c|c|c|c|c|c|}
\hline SITUACIÓN & $\begin{array}{l}\text { ALTO } \\
\text { RIESGO }\end{array}$ & $\begin{array}{l}\text { REGULAR } \\
\text { RIESGO }\end{array}$ & $\begin{array}{l}\text { BAJO } \\
\text { RIESGO }\end{array}$ & $\begin{array}{l}\text { NO HAY } \\
\text { RIESGO }\end{array}$ & TOTAL \\
\hline $\begin{array}{l}\text { Relación coital con } \\
\text { preservativo con VIH }\end{array}$ & 94,5 & 3,1 & 2,0 & 0,4 & 100,0 \\
\hline $\begin{array}{l}\text { Jeringa o aguja contaminada } \\
\text { por otros }\end{array}$ & 94,5 & 2,0 & 0,8 & 2,7 & 100,0 \\
\hline $\begin{array}{l}\text { Relación coital con personas } \\
\text { del otro sexo sin usar } \\
\text { preservativo }\end{array}$ & 86,7 & 8,4 & 4,5 & 0,4 & 100,0 \\
\hline $\begin{array}{l}\text { Relación coital con personas } \\
\text { del mismo sexo sin usar } \\
\text { preservativo }\end{array}$ & 81,7 & 15,2 & 2,5 & 0,6 & 100,0 \\
\hline $\begin{array}{l}\text { Practicar sexo anal sin usar } \\
\text { preservativo }\end{array}$ & 83,7 & 12,0 & 3,4 & 0,9 & 100,0 \\
\hline $\begin{array}{l}\text { Relación coital con personas } \\
\text { del mismo sexo con } \\
\text { preservativo }\end{array}$ & 78,1 & 17,0 & 3,4 & 1,5 & 100,0 \\
\hline $\begin{array}{l}\text { Practicar sexo oral con } \\
\text { cualquier otra persona }\end{array}$ & 45,8 & 31,4 & 18,1 & 4,8 & 100,0 \\
\hline $\begin{array}{l}\text { Relación coital sin usar } \\
\text { preservativo con persona con } \\
\text { VIH }\end{array}$ & 34,0 & 39,1 & 25,3 & 1,7 & 100,0 \\
\hline $\begin{array}{l}\text { Recibir una transfusión de } \\
\text { sangre }\end{array}$ & 31,5 & 26,6 & 26,4 & 15,5 & 100,0 \\
\hline $\begin{array}{l}\text { Practicar sexo anal usando el } \\
\text { preservativo }\end{array}$ & 16,8 & 41,3 & 36,9 & 5,0 & 100,0 \\
\hline $\begin{array}{l}\text { Relación coital con personas } \\
\text { de otro sexo usando } \\
\text { preservativo }\end{array}$ & 10,0 & 28,7 & 56,4 & 4,9 & 100,0 \\
\hline $\begin{array}{l}\text { Asistir a clínica dental } \\
\text { o centro de salud donde } \\
\text { atienden personas con sida }\end{array}$ & 9,0 & 18,5 & 38,2 & 34,3 & 100,0 \\
\hline Donar sangre & 8,1 & 13,9 & 23,3 & 54,6 & 100,0 \\
\hline Picadura de un mosquito & 7,2 & 6,7 & 16,4 & 69,7 & 100,0 \\
\hline Jugar con niño que tiene sida & 3,4 & 0,4 & 4,3 & 91,9 & 100,0 \\
\hline $\begin{array}{l}\text { Usando preservativo cada vez } \\
\text { que se tiene relación sexual }\end{array}$ & 3,0 & 19,9 & 58,3 & 18,9 & 100,0 \\
\hline $\begin{array}{l}\text { Tener relaciones sexuales con } \\
\text { única pareja fiel no infectada }\end{array}$ & 1,4 & 4,1 & 19,4 & 75,1 & 100,0 \\
\hline Nadar en una piscina pública & 0,2 & 0,9 & 6,8 & 92,1 & 100,0 \\
\hline Usar un inodoro público & 0,0 & 4,5 & 17,4 & 78,1 & 100,0 \\
\hline $\begin{array}{l}\text { Dar la mano o besar a alguien } \\
\text { que tiene sida }\end{array}$ & 0,0 & 1,0 & 3,9 & 95,1 & 100,0 \\
\hline
\end{tabular}


CUADRO 2

UNIVERSIDAD DE COSTA RICA: DISTRIBUCIÓN DE LOS ESTUDIANTES

ENTREVISTADOS ANTE ASEVERACIONES SOBRE EL SIDA, 2008

\begin{tabular}{|c|c|c|c|}
\hline ASEVERACIONES & DE ACUERDO & EN DESACUERDO & TOTAL \\
\hline $\begin{array}{l}\text { Detección del sida puede ser negativa días } \\
\text { después de tener relación con persona } \\
\text { infectada }\end{array}$ & 72,2 & 27,8 & 100,0 \\
\hline $\begin{array}{l}\text { Mayor riesgo de contagio de sida por sexo } \\
\text { anal sin protección }\end{array}$ & 66,1 & 33,9 & 100,0 \\
\hline Con esto del sida nadie está a salvo & 35,3 & 64,7 & 100,0 \\
\hline $\begin{array}{l}\text { El VIH no se transmite por uso de objetos } \\
\text { contaminados }\end{array}$ & 26,4 & 73,6 & 100,0 \\
\hline $\begin{array}{l}\text { El sida es una enfermedad, no debemos } \\
\text { cambiar }\end{array}$ & 8,7 & 91,3 & 100,0 \\
\hline $\begin{array}{l}\text { Es poco lo que puede hacerse para evitar } \\
\text { el sida }\end{array}$ & 7,7 & 92,3 & 100,0 \\
\hline $\begin{array}{l}\text { El sida puede controlarse obligando a } \\
\text { homosexuales a hacerse la detección }\end{array}$ & 5,9 & 94,1 & 100,0 \\
\hline El sida solo les da a los homosexuales & 5,6 & 94,4 & 100,0 \\
\hline Existe una vacuna para prevenir el sida & 5,5 & 94,5 & 100,0 \\
\hline Tanto ruido por el sida es exagerado. & 5,3 & 94,7 & 100,0 \\
\hline $\begin{array}{l}\text { El sida debe preocupar solo a los } \\
\text { homosexuales. }\end{array}$ & 3,2 & 96,8 & 100,0 \\
\hline $\begin{array}{l}\text { Pocos casos en Costa Rica, difícil } \\
\text { contagiarse. }\end{array}$ & 2,6 & 97,4 & 100,0 \\
\hline
\end{tabular}


CUADRO 3

UNIVERSIDAD DE COSTA RICA: PROPORCIÓN DE ESTUDIANTES QUE RESPONDIERON QUE ES DE ALTO O REGULAR RIESGO EL CONTAGIARSE DE VIH-SIDA EN DISTINTAS SITUACIONES POR SEXO, 2008

\begin{tabular}{|c|c|c|}
\hline CLASIFICACIÓN Y SITUACIONES & HOMBRE & MUJER \\
\hline \multicolumn{3}{|l|}{ ESTEREOTIPO } \\
\hline Usar un inodoro público. & 3,1 & 8,2 \\
\hline $\begin{array}{l}\text { Dar mano o besar en la mejilla a alguien } \\
\text { con sida. }\end{array}$ & 0,9 & 1,1 \\
\hline Nadar en una piscina pública. & 1,0 & 1,2 \\
\hline Jugar con un niño que tiene sida. & 5,7 & 2,4 \\
\hline Asistir a una clínica dental o centro de & & \\
\hline salud donde atiende personas con sida. & 23,0 & 31,6 \\
\hline Picadura de un mosquito. & 15,6 & 12,3 \\
\hline $\begin{array}{l}\text { Tener relaciones sexuales con única } \\
\text { pareja fiel no infectada. }\end{array}$ & 6,0 & 4,9 \\
\hline \multicolumn{3}{|l|}{ CONOCIMIENTOS } \\
\hline $\begin{array}{l}\text { Usar jeringa o aguja contaminada usada } \\
\text { por otros. }\end{array}$ & 95,0 & 97,8 \\
\hline Recibir una transfusión de sangre. & 50,7 & 64,8 \\
\hline Donar sangre. & 22,0 & 22,2 \\
\hline \multicolumn{3}{|l|}{ PRÁCTICAS SEXUALES CON } \\
\hline $\begin{array}{l}\text { Relación coital con persona del otro sexo } \\
\text { con preservativo. }\end{array}$ & 36,3 & 40,9 \\
\hline $\begin{array}{l}\text { Relación coital con preservativo con } \\
\text { persona que tiene VIH. }\end{array}$ & 72,7 & 73,7 \\
\hline $\begin{array}{l}\text { Relación coital con persona del mismo } \\
\text { sexo con preservativo. }\end{array}$ & 95,0 & 95,2 \\
\hline Practicar sexo anal con preservativo. & 56,2 & 59,6 \\
\hline $\begin{array}{l}\text { Usando preservativo cada vez que tiene } \\
\text { relación sexual. }\end{array}$ & 55,8 & 23,0 \\
\hline \multicolumn{3}{|l|}{ PRÁCTICAS SEXUALES SIN } \\
\hline $\begin{array}{l}\text { Relación coital con persona del otro sexo } \\
\text { sin preservativo. }\end{array}$ & 92,8 & 97,0 \\
\hline $\begin{array}{l}\text { Relación coital sin preservativo con } \\
\text { persona que tiene VIH. }\end{array}$ & 95,9 & 98,9 \\
\hline $\begin{array}{l}\text { Relación coital con persona del mismo } \\
\text { sexo sin preservativo. }\end{array}$ & 98,2 & 95,7 \\
\hline Practicar sexo anal sin preservativo. & 95,4 & 96,0 \\
\hline PRACTICA SEXO ORAL & & \\
\hline Practicar sexo oral con cualquier persona. & 72,7 & 81,0 \\
\hline
\end{tabular}


CUADRO 4

UNIVERSIDAD DE COSTA RICA

VALOR DE LA MEDIANA, PROMEDIO Y DESVIACIÓN ESTÁNDAR DE LA NOTA

EN CONOCIMIENTOS POR SEXO Y EDAD, 2008

\begin{tabular}{lcccc}
\hline SEXO Y EDADES & $(\mathrm{n})$ & MEDIANA & MEDIA & DESV. TIP. \\
\hline Total & 497 & 62,5 & 65,5 & 18,6 \\
Hombre & 224 & 62,5 & 64,7 & 19,3 \\
Mujer & 271 & 62,5 & 66,4 & 17,4 \\
17 a 19 años & 97 & 62,5 & 64,0 & 17,7 \\
20 a 22 años & 218 & 62,5 & 64,5 & 19,6 \\
23 años y más. & 172 & 75,0 & 68,6 & 16,2 \\
& & & & \\
\hline
\end{tabular}

Fecha de ingreso: $12 / 01 / 2011$

Fecha de aprobación: 31/06/2011 